\title{
UCRL-TR-231783
}

LAW RENCE LIVERMORE NATIO N A L LABORATORY
Evaluation of Polymer-Filler Interaction Characteristics by Force Microscopy

T.V. Ratto, A. Saab

June 14, 2007 
This document was prepared as an account of work sponsored by an agency of the United States Government. Neither the United States Government nor the University of California nor any of their employees, makes any warranty, express or implied, or assumes any legal liability or responsibility for the accuracy, completeness, or usefulness of any information, apparatus, product, or process disclosed, or represents that its use would not infringe privately owned rights. Reference herein to any specific commercial product, process, or service by trade name, trademark, manufacturer, or otherwise, does not necessarily constitute or imply its endorsement, recommendation, or favoring by the United States Government or the University of California. The views and opinions of authors expressed herein do not necessarily state or reflect those of the United States Government or the University of California, and shall not be used for advertising or product endorsement purposes.

This work was performed under the auspices of the U.S. Department of Energy by University of California, Lawrence Livermore National Laboratory under Contract W-7405-Eng-48. 


\section{Evaluation of Polymer-Filler Interaction Characteristics by Force Microscopy.}

Timothy Ratto

Andrew P. Saab

Evaluation of Polymer-Filler Interaction Characteristics by Force Microscopy __ 1

Motivation__ 2

Atomic Force Microscopy: Fundamentals _ 2

Force spectroscopy- Normal mode __ 4

Force Spectroscopy- Lateral mode__ 6

Calibration 6

Method 1:

Method 2:

Lateral Force Measurements:______ 9

Converting Force Measurements To Material Properties __ 11

Sample preparation ___ 13

Experiments: 14

Results from imaging___ 14

Force Measurements ——a 17

Future Work: _ 23

References__ 23

Appendix 1: Normal -mode calibration 


\section{Motivation}

Silicone polymers are frequently used as cushions and inserts between load bearing parts. In this capacity, they must act to position their associated parts and distribute mechanical force as appropriate. One type of failure is specific to silicones that are filled with high surface area particulates for purposes of tailoring the polymer compressive properties. Additives such as fumed silicon oxide are presumed to have a high degree of surface interaction with the polymer matrix, thus causing the polymer to stiffen and to display greater dimensional stability as a function of temperature. However, it has been observed that the compressive behavior of these materials is not always invariant over long times. There is evidence that suggests changes in humidity and temperature can irreversibly alter the silicone-filler interaction, thereby changing the overall characteristics of parts made from such materials. As before, changes in compressive or shear stability can have serious effects on the ability of these materials to effectively position precision parts or distribute high mechanical loads.

We approach the analysis of the filled systems by creating controlled layers of silicone polymers attached to silicon oxide substrates. Straight chain vinyl-silicone polymers identical to those used in the formulation of pads for stockpile systems are chemically appended to a substrate surface, and cross-linked to form a three dimensional network. This type of structure serves as a model of silicone polymer coating a silicon oxide filler particle.

We study these model systems first by using Atomic Force Microscopy (AFM) to image the samples with nanometer resolution, and then by measuring the forces of interactions between single model silica filler particles and polymer-coated surfaces. We use normal longitudinal force AFM to measure adhesion, and a relatively newly developed technique, lateral force AFM, to determine the frictional forces between the silica particles and the polymer films. Lateral force AFM is a sophisticated technique that involves observing the torsional deflections of a cantilever that is scanned across a surface perpendicular to the normal mode deflection. For a carefully calibrated system, this gives information on the dynamic frictional component of the particle/polymer interaction. Both force-measuring techniques utilize colloidal silicon oxide probes ranging from $0.6 \mu \mathrm{m}$ to $2.0 \mu \mathrm{m}$ in diameter. These probes replace the standard sharp AFM tip on the cantilever with a spherical bead (Figure 1) and are used to examine interactions between the bead material and the sample surface.

Atomic Force Microscopy: Fundamentals In its most common form Atomic Force Microscopy (AFM) is a technique that drags a micromachined stylus or tip across a sample surface and produces topographic images of the sample ${ }^{1}$. As this technique operates in the near-field (versus the far field as does optical microscopy) the resolution is therefore limited only to the size of the AFM tip. In addition to physical topology however, AFM can be used to produce images or data which contain other types of surface information (e.g. the distribution of charge, magnetic domains, nanoscale chemical modifications, etc.). In order to recognize the potential of this technique it is important to consider AFM as a technique whereby a probe is used to carry out a local experiment at any point where the probe is in contact with the surface. The results of all 
successive experiments are collected and reported as a function of the probe's position on the sample. In this way, an image produced by AFM can contain information about the sample from the viewpoint of the explicitly chosen experimental parameters. Consequently, different operational parameters (e.g. chemical modification of the probe) will result in images which contain information about the scanned surface. In addition, scanning probe devices can often be operated in single point or line mode (as we discuss below in Force Spectroscopy) in order to increase the fidelity of the experiment that is conducted on the local scale.

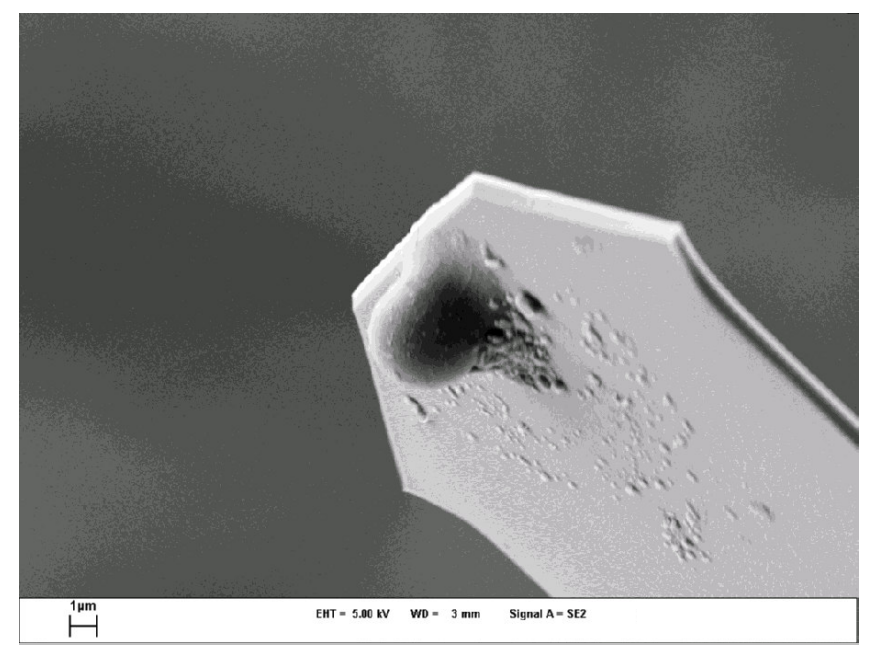

Figure 1. This SEM image shows a 5 micron (um) spherical silica bead attached to a rectangular AFM cantilever. These colloidal probes are often used to examine adhesive and frictional interactions between the bead and a sample surface.

For the measurements discussed in this report, we use a Veeco Instruments Multimode Atomic Force Microscope (AFM) controlled by a Nanoscope III controller. The central component of this device is the microcantilever, which bears either a sharp probe that is usually between 5-30 $\mathrm{nm}$ at the tip, or a colloidal probe as shown above. When the tip interacts locally with the sample surface, the force of interaction causes the cantilever to bend. A laser is deflected from the cantilever and impinges on a quadrant photodetector (Figure 2). Deflection of the cantilever in the normal or $\mathrm{Z}$ direction is measured by subtracting the voltage from the top two quadrants from the bottom two. Similarly, lateral deflection is measured by subtracting the right two quadrants from the left (Figure 3). In order to map out the topography of a surface in contact with the tip (contact mode imaging) a feedback circuit attempts to maintain a constant deflection of the cantilever (i.e. a constant force) by raising and lowering the lever using a piezoelectric scanner. The voltage required to move the lever is mapped as a function of the xy position of the lever and produces a contact mode image. Another method for generating images of a sample surface uses the damping of the cantilever oscillation as a feedback mechanism. In this mode (called tapping or noncontact mode) the cantilever is driven at its natural resonance frequency and the magnitude of the resonance is measured by the photodetector. 


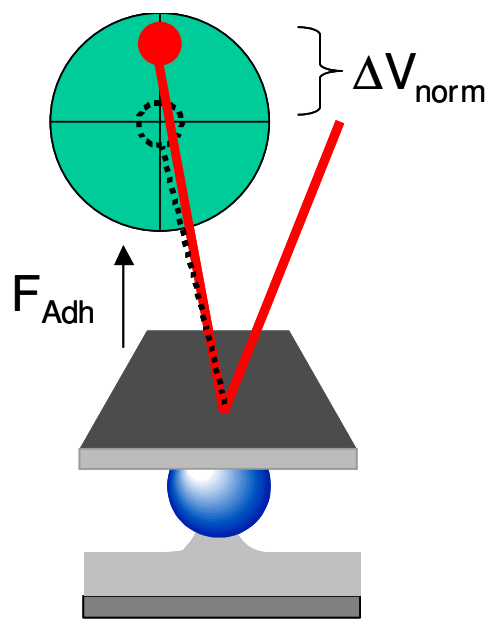

Figure 2. The AFM operates by moving a cantilever bearing a sharp tip into contact with a substrate. Deflections of the cantilever are detected in a photodetector by measuring the movement of a laser beam reflected from the cantilever. In this schematic, the adhesion force between a spherical tip and a sample is being measured at a single point. Raster scanning the tip across the sample allows the mapping of local features and produces an image whereby the resolution is limited only by the size of the AFM tip.

When the tip is close to (or in contact with) the sample surface the magnitude of the tip resonance is damped. Similarly to contact mode, the feedback circuit attempts to maintain a constant oscillation magnitude by raising and lowering the cantilever and the piezoelectric scanner voltage is used to produce an image. Due to the lowered degree of tip-sample contact, tapping or noncontact mode is often used to image soft samples to prevent damage by the tip.
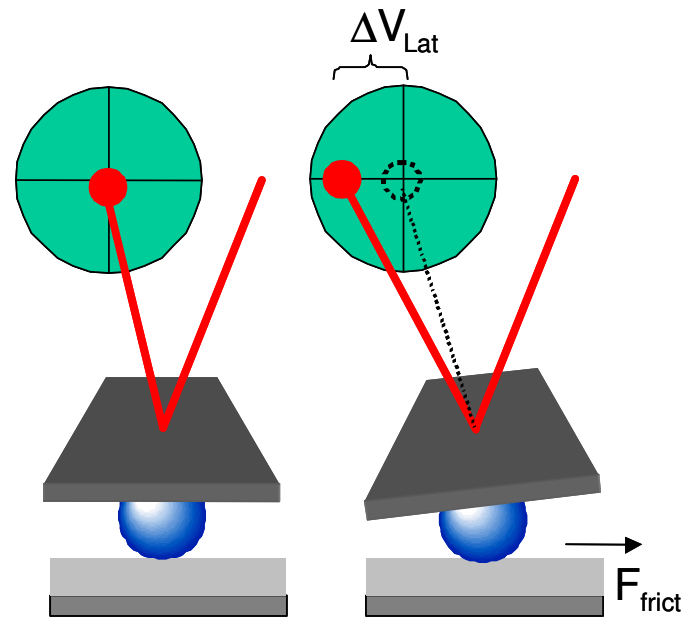

Figure 3. To measure lateral or frictional forces between a tip and substrate the AFM can be operated in lateral mode whereby the twist of the cantilever due to friction between the tip and sample is measured.

Force spectroscopy- Normal mode Normal mode force spectroscopy allows the measurement of forces that are applied along the $\mathrm{Z}$-axis of the cantilever ${ }^{2-7}$. Typical measurements include nanoscale adhesion and the elastic response of thin films. The AFM device does not in itself directly measure force, rather it emulates a spring and Hooke's law is used to calculate the force applied to the lever as:

$$
F_{z}=k_{z} z
$$

where $k_{z}$ and $z$ are the spring constant and the deflection of the cantilever respectively and $\mathrm{F}_{\mathrm{z}}$ is the measured force.

Note that in this case we are ignoring the contribution to the force due to the elasticity of the material in contact with the AFM cantilever (the contact stiffness) which 
would require us to model the system as two springs in series rather than as a single spring. In most cases an appropriate cantilever can be selected that has a much lower spring constant than almost all polymer samples, allowing us to disregard the contact stiffness. This is not the case however for lateral force measurements (as shown in the next section) where the lever is much stiffer in torsion as opposed to bending (it is harder to twist a beam than it is to bend it.) Lateral force measurements require treating the lever-sample system like two springs in series adding a considerable amount of difficulty to the calibration of the measurement.

The AFM accurately measures two quantities, the distance the cantilever is moved by the piezoelectric positioner $\mathrm{Z}_{\mathrm{PD}}$, and the deflection of the laser spot on the detector in Volts. In order to use Hooke's law to calculate the force, the detector calibration factor must be determined. This parameter translates the deflection of the laser spot on the detector in (volts) to a physical deflection of the lever (in nanometers). This factor, commonly called the sensitivity of the detector, $\zeta$, is ascertained by moving the cantilever into contact with a flat substrate so that a movement in $\mathrm{z}$ corresponds directly to a deflection of the cantilever. The slope of this line in V/nm is the sensitivity of the detector and permits the calculation of the physical defection of the cantilever as:

$$
z=z_{P D} \zeta
$$

where $\mathrm{Z}_{\mathrm{L}}$ is the physical deflection of the cantilever, $\mathrm{Z}_{\mathrm{PD}}$ is the deflection of the laser spot on the photodetector due to a deflection of the cantilever, and $\zeta$ is the sensitivity of the detector.

The calculation of the force applied to the cantilever also requires the spring constant, $\mathrm{k}_{\mathrm{z}}$ of the cantilever. For a rectangular lever the spring constant can be calculated as ${ }^{8}$ :

$$
k_{z}=\frac{4 l^{3}}{E w t^{3}}
$$

where $\mathrm{E}$ is the elastic modulus of the cantilever and $1, \mathrm{w}$, and $\mathrm{t}$ are the length, width and thickness.

Unfortunately the thickness of AFM cantilevers is a challenging parameter to accurately determine, as it is controlled by the microfabrication process (in fact the thickness of the lever is determined by the final etch, a notoriously inconsistent step) and can vary considerably from lever to lever. As thickness $(t)$ is cubed in $k_{z}$ even small errors in this determination lead to quite large errors in the spring constant.

A more accurate method for determining the cantilever spring constant uses the equipartition theorem to relate the measured thermal noise in the AFM cantilever deflection signal to the spring constant in the cantilever, which is assumed to behave like an ideal spring (a simple harmonic oscillator) ${ }^{3,9,10}$. The equipartition theorem explicitly states that the thermal energy present in all terms in the Hamiltonian of a system that are quadratically dependent on a generalized coordinate is equal to $k_{\mathrm{B}} T / 2$, where $k_{\mathrm{B}}$ is Boltzmann's constant and $T$ is the absolute temperature (in Kelvin) thus: 


$$
\frac{1}{2} k_{z}\left\langle x^{2}\right\rangle=\frac{1}{2} K_{B} T
$$

and

$$
k_{z}=\frac{K_{B} T}{\left\langle x^{2}\right\rangle}
$$

The spring constant calibration therefore requires only the ability to measure the temperature and the average deflection of the cantilever in the time domain (in order to exclude other sources of noise, such as electronic noise, $60 \mathrm{~Hz}$ noise, table vibrations, etc., the noise is measured in the frequency domain).

Force Spectroscopy- Lateral mode Lateral mode force spectroscopy permits the measurement of forces that are applied transversely to the length of the cantilever ${ }^{11-15}$. Data from a lateral force experiment is shown in Figure 4. To produce this data an AFM cantilever is moved laterally back and forth across a flat sample and the lateral deflection of the cantilever is measured by a photodetector. When the cantilever first begins its lateral motion, the tip sticks to the substrate and the cantilever undergoes a lateral deflection or twist. When the lateral force on the cantilever increases beyond the static friction coefficient, the tip slips along the substrate until it reaches the end of its travel at which point its motion is reversed. The two traces (forward and back) are combined into a single "friction loop" (Figure 4), The voltage difference between the two traces contains information regarding the tip-sample frictional interaction.

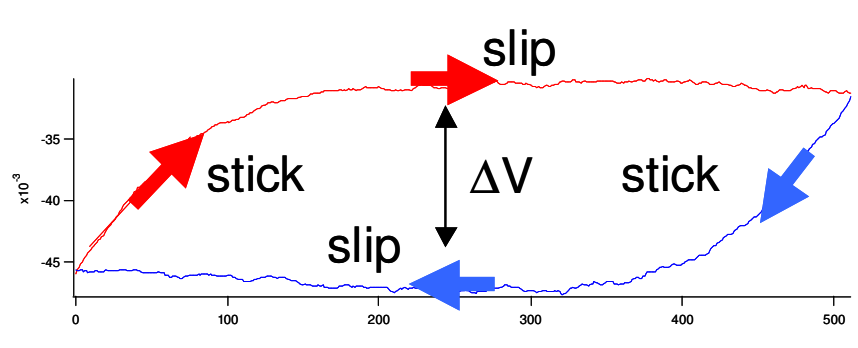

Figure 4. Friction loop showing the stick-slip behavior of the AFM tip as it moves laterally forward and back across a sample surface. The y-axis is the lateral deflection or twist of the cantilever. The $\mathrm{x}$-axis is the distance traveled by the tip.

Calibration Lateral force mode (LFM) is used mainly to measure frictional forces between a tip-bearing cantilever and the substrate. Although there are many similarities between normal force and lateral force modes there are some important differences that require additional effort in order to properly make these measurements. Most significantly, wherein with the normal force measurements the elastic response of the tipmaterial contact can be disregarded in the calculation due to the magnitude of the contact stiffness, $\mathrm{k}_{\mathrm{c}}$ (i.e. $\mathrm{k}_{\mathrm{cs}}>>\mathrm{k}_{\mathrm{z}}$ ) for most frictional measurements the cantilever lateral spring constant and the contact stiffness are comparable in magnitude (i.e. $\mathrm{k}_{\mathrm{L}} \sim \mathrm{k}_{\mathrm{cs}}$ ) and thus the system must be treated as two springs in series ${ }^{16}$. The force and deflection due to the force may be modeled as follows, where $\mathrm{F}_{\mathrm{L}}$ is the friction force necessary to cause slip at the contact interface, therefore:

$$
F_{L}=k_{y c} y_{c}=k_{y} y=k_{t o t} y_{s}
$$




$$
k_{t o t}=\left[\frac{1}{k_{y}}+\frac{1}{k_{y c}}\right]^{1 / 2}
$$

Where $\mathrm{y}_{\mathrm{s}}$ is the lateral scanner travel, $\mathrm{y}_{\mathrm{c}}$ is the lateral deformation of the contact, and $\mathrm{y}$ is the component of the lateral motion at the interface leading to the twisting of the cantilever. $\mathrm{k}_{\mathrm{tot}}$ is the combined spring constant of the cantilever and the tip-sample contact.

In terms of the combined motion of the cantilever, the scanner, and the tip-sample contact:

$$
y_{s}=y+y_{c}
$$

The twist of the cantilever is detected by the photodetector which outputs a voltage that is proportional to the torsional motion of the lever:

$$
V_{L F M}=\zeta \phi
$$

Where $\zeta$ is the lateral detector sensitivity (this value is analogous to the normal sensitivity (s) for normal mode force measurements discussed above) and $\varphi$ is the physical twist in radians of the cantilever.

The slope, S, of the LFM output while the tip sticks to the sample is therefore equal to the twist in the cantilever divided by the scanner travel $\mathrm{y}_{\mathrm{s}}$. Therefore:

$$
S=\frac{V_{L F M}}{y_{s}}=\frac{\zeta \phi}{y_{s}}
$$

And since the twist in the cantilever in radians is equal to the lateral component of motion, $y$, divided by the torque arm distance from the cantilever to the sample surface (this is essentially the tip height, $h$ ) thus:

$$
\begin{aligned}
& \phi=y / h \\
& h S=\frac{y \zeta}{y_{s}}
\end{aligned}
$$

Remembering that $\mathrm{k}_{\mathrm{tot}} \mathrm{y}_{\mathrm{s}}=\mathrm{k}_{\mathrm{y}} \mathrm{y}$ :

$$
h S=\frac{k_{t o t} \zeta}{k_{y}}
$$

For this method, the lateral detector sensitivity, $\zeta$, and the lateral cantilever spring constant are assumed to be constant, so that hS varies only with the combined stiffness $\mathrm{k}_{\mathrm{tot}}$. If a condition can be found in which all of the deflection is occurring in the cantilever then $\mathrm{k}_{\mathrm{tot}}=\mathrm{k}_{\mathrm{y}}$ and $\mathrm{hS}=\zeta^{16}$. From experiment this condition is met with large-radii 
cantilever tips ( $\geq 1$ micron) at loads greater than $2000 \mathrm{nN}$. Before starting to take data therefore, the detector lateral sensitivity, $\zeta$, can be obtained by generating friction loops at high load forces and then measuring the slope, $S$, of the trace at the points where the cantilever is twisting. Multiplying by the height, $\mathrm{h}$, of the tip from the cantilever produces the sensitivity value. For cantilevers with attached colloidal spheres the height is calculated as $h=2 R+t$, where $R$ is the radius of the attached sphere and $t$ is the thickness of the cantilever.

Once the lateral detector sensitivity is obtained, the lateral spring constant, $\mathrm{k}_{\mathrm{L}}$, of the cantilever must be acquired. We use two different methods to measure $\mathrm{k}_{\mathrm{L}}$

Method 1: The first relies on published simulations of the mechanical behavior of a rectangular lever under an applied load ${ }^{17,18}$. Although the simulations do not produce an analytical solution, numerical methods are used to approximate the resulting bending behavior of the cantilever. This method is similar to the normal mode spring constant $\left(\mathrm{k}_{\mathrm{z}}\right)$ calibration in that it requires a measurement of the resonance frequency of the cantilever. In this case however, it is the torsional resonance (rather than the normal mode) that is required. Assuming that the length of a cantilever, $\mathrm{L}$, is much greater than its width, $b$, which in turn greatly exceeds its thickness, $t$, than the torsional spring constant, $k_{\phi}$, is given by:

$$
k_{\phi}=\frac{1}{3 \pi^{2}} \rho_{c} b^{3} t L \omega_{t, v a c}^{2}
$$

Where $\rho_{\mathrm{c}}$ is the density of the cantilever and $\omega_{t, v a c}$ is the resonant frequency of torsional vibration in a vacuum.

This equation is of limited use however, due to the difficulty in measuring cantilever density and thickness, and the resonant frequency in a vacuum. From recently published theoretical models for cantilever beams immersed in viscous fluids ${ }^{17,18}$ it has been determined that the vacuum resonant frequency can be directly related to the resonant frequency in air or fluid, $\omega_{\mathrm{t}}$ by:

$$
\omega_{t, v a c}=\omega_{t}\left(1+\frac{3 \pi \rho b}{2 \rho_{c} t} \Gamma_{r}^{t}\left(\omega_{t}\right)\right)^{1 / 2}
$$

Where $\rho$ is the density of the gas or fluid and $\Gamma_{r}^{t}\left(\omega_{t}\right)$ is the real part of the hydrodynamic function for a cantilever which depends only on the Reynolds number, Re.

In addition the mass density of the cantilever $\left(\rho_{c} h\right)$ is only dependent on the density of the surrounding medium, the width of the cantilever, and the quality factor, Q. Substituting these in to the equation for $\mathrm{k}_{\phi}$ we find:

$$
k_{\phi}=0.1592 \rho b^{4} L Q \omega_{t} \Gamma_{r}^{t}\left(\omega_{t}\right)
$$

This result relates the torsional spring constant at the end of the cantilever to its dimensions, L,b, the resonant peak and Q value in air of fluid, and the density of the air 
or fluid. The hydrodynamic function, $\Gamma$, can be obtained from a look-up table. Finally the lateral spring constant can be calculated from the torsional spring constant, $\mathrm{k}_{\phi}$, as:

$$
k_{L}=\frac{k_{\phi}}{h^{2}}
$$

Where $\mathrm{h}$ is the distance between the sample surface and the top of the cantilever and for a bead attached to a cantilever is given by twice the radius of the bead.

Method 2: The second method measures the lateral twist of the cantilever directly by utilizing a calibration substrate that displays planes which are tilted at known degrees (Figure 5) ${ }^{19}$. This technique has the advantage of providing a calibration factor, $\alpha$, that contains both the detector sensitivity and the lateral spring constant in one constant. That is $\alpha$, in Newtons/Volt provides a direct translation from the voltage measured on the detector to the lateral force encountered by the AFM cantilever. When the cantilever is dragged laterally across the calibration substrate the twist of the cantilever in response to the lateral applied force (simply a function of the applied force in the z-direction) is measured. The torsional force acting on the cantilever due to a tilt in the substrate is then compared to the torsion due to friction on a flat substrate. Although this method can generate highly reliable spring constants, the actual measurements required to deliver precise and reproducible results are tedious and time consuming. We have begun the process of automating this mode of calibration by producing custom LabView (National Instruments) code that interfaces directly with the AFM and will simplify the process.
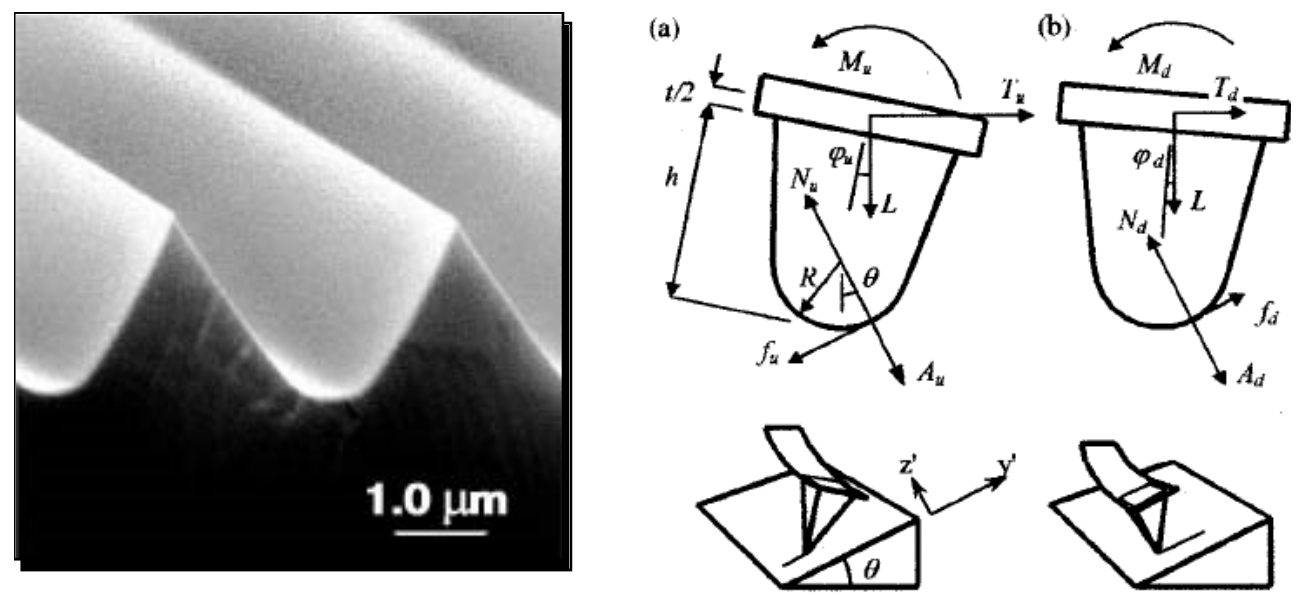

Figure 5. An SEM image of a lateral force calibration grid and a schematic of the geometry used to relate the measured lateral deflection to a lateral force.

Lateral Force Measurements: Finally, once the detector sensitivity and the lateral spring constant are obtained, the lateral force can be calculated as:

$$
F_{L}=\frac{k_{L} \Delta V_{L}}{2 R \zeta_{L}}
$$




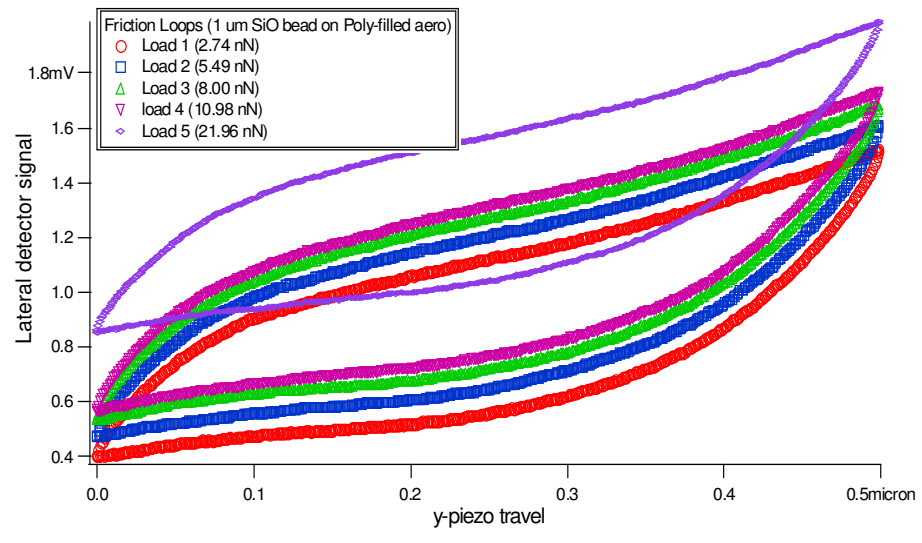

Figure 6. Friction loops measured between a 1.0 micron silicon oxide bead and a polymer-coated aerogel surface. Each loop is taken at a different load force and produces a single lateral force measurement, $\mathrm{F}_{\mathrm{L}}$.

where $\Delta \mathrm{V}_{\mathrm{L}}$ is measured as the difference between the forward and backward lateral deflection traces in the friction loop (see Figure 4).

To perform an experiment, friction loops are obtained at increasing load forces (Figure 6) and the lateral force, $\mathrm{F}_{\mathrm{L}}$, is calculated. The lateral force versus the load force is then graphed (Figure 7). Model fits to this data allow the extraction of physical properties of the tip-sample interaction as described in the next section. Initial measurements of the lateral force as a function of loading force between a 1.0 micron silicon oxide bead attached to the cantilever and a polymer coated aerogel surface showed an unexpected behavior. As shown in Figure 7, the friction force did not increase with the loading force as expected.

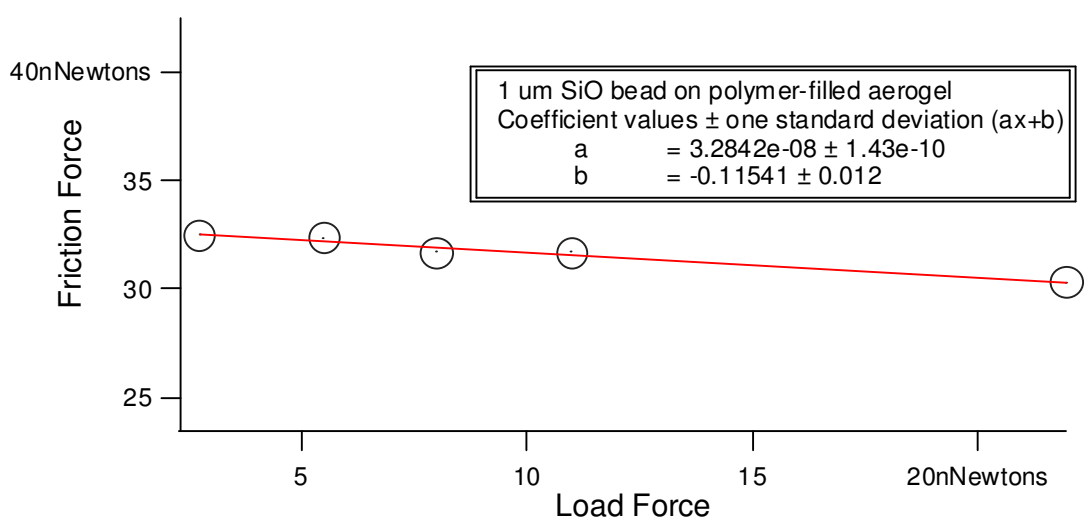

Figure 7. Friction vs load force for a 1.0 um silicon oxide bead on a polymer coated aerogel surface. Unexpectedly, the friction force decreased with increased loading force.

We discovered that the detector sensitivity was to blame. Rather than being constant with loading force as predicted, at increased loading forces the detector sensitivity decreases. This can be explained first by reviewing the assumptions that are made in measuring the detector voltage. The first, that the laser spot on the detector is round (see Figure 1) is false ${ }^{20}$. Rather, the spot is non-axially symmetric so that as the spot moves up the detector due to increasing cantilever deflections (as a consequence of higher loading forces) the 
detector output voltage does not increase linearly with deflection. Additionally, it appears that the sensitivity of the detector itself is not uniform across the entire surface, leading to errors at higher cantilever deflections. We compensated for this effect by measuring the detector sensitivity at the loading forces used in the experiments and constructing a load calibration graph (Figure 8). Including the load calibrations with the lateral force data produced the correct lateral force versus loading force behavior (Figure 9).

Converting Force Measurements To Material Properties Force measurements alone- while useful for determining the amount of force required to break a bond to a surface or predicting the lifetime of a component undergoing friction- do not in themselves reveal underlying material properties of the sample under study. In fact, the forces measured using the AFM under proper conditions are assumed to be singleasperity elastic contact forces, and are directly dependent on the physical area of the contact $^{11,21}$. Continuum models that include a prediction of the contact geometry are therefore required to extract material properties of the samples undergoing study. Three analytical models are commonly used, the Hertz, the Johnson-Kendall-Roberts (JKR), and the Derjaguin-Muller-Toporov (DMT). In the absence of adhesion, the Hertz model has been shown to accurately predict the material properties of interacting elastic spheres.

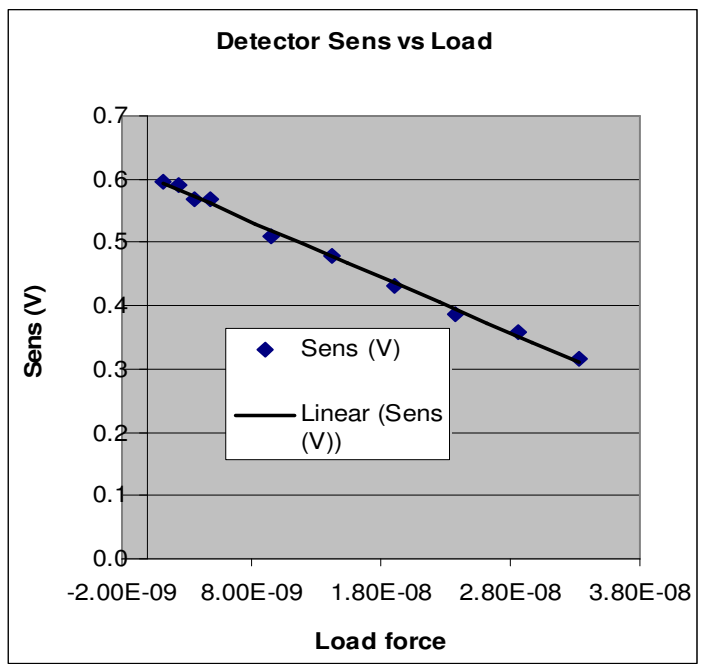

Figure 8. AFM detector sensitivity as a function of the applied loading force. As shown, the detector sensitivity decreases as the laser spot moves away from the center of the detector. This can be explained in two ways, firstly, the laser spot is not axially symmetric on the detector, and secondly, the light sensitivity is not uniform across the detector surface.

However, at the small scales typically probed by AFM the surface-to-bulk ratio (the relative amount of interacting surface area versus the interacting bulk material) becomes significant and adhesion can not be discounted ${ }^{22}$. The two models that include adhesion, the JKR and DMT, are essentially limiting cases for the spatial ranges over which the measured forces operate. When the surface forces are short range in comparison to the elastic deformations they cause (i.e. compliant materials, strong adhesion, large tip radii), the JKR effectively predicts the area of contact and thus the material properties of the sample. When the reverse is true (i.e. stiff materials, weak adhesion, small tip radii) the DMT applies. Rather than making assumptions regarding which model applies to the current system under study, a process is used to fit the experimentally measured friction data which then reveals the appropriate model. This process is as follows, the variation of contact area with load can be generalized to a single equation: 


$$
a=a_{0}\left(\frac{\alpha+\sqrt{1-L / L_{c(\alpha)}}}{1+\alpha}\right)
$$

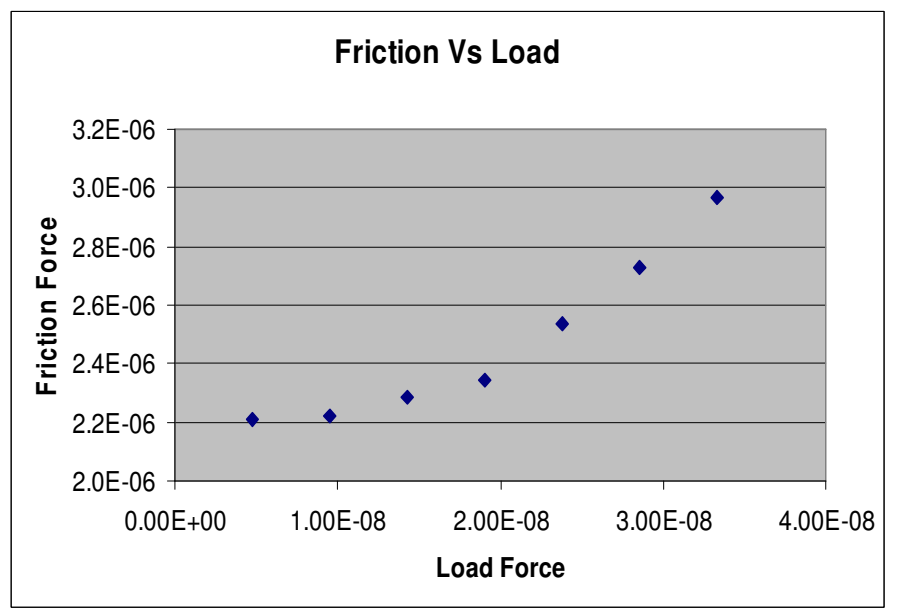

Figure 9. As predicted for single asperity contacts, the friction force between a 1 micron silicon oxide bead and a polymercoated aerogel surface increases with increasing loading forces.

Unfortunately, the aerogel surface proved to be too rough on the nanoscale to make reproducible lateral force measurements.

where $\mathrm{a}$ is the contact radius, $\mathrm{a}_{0}$ is the contact radius at zero load, $\mathrm{L}$ is the load force, and $\mathrm{L}_{\mathrm{c}(\alpha)}$ is the negative critical load (or measured pull-off force) and is related directly to the adhesion between the tip and sample. $\alpha$ is a model dependent parameter and equals 1 for the JKR case, and 0 for the DMT.

Assuming that friction is directly proportional to the contact area:

$$
F_{L}=\tau \cdot \pi a^{2}
$$

where $F_{L}$ is the measured lateral force, $\tau$ is the interfacial shear stress, and a is the contact radius.

Combining the two equations:

$$
F_{L}=\tau \pi a_{0}^{2}\left(\frac{\alpha+\sqrt{1-L / L_{c(\alpha)}}}{1+\alpha}\right)^{2 / 3}
$$

Fitting the experimental data (lateral force, $\mathrm{F}_{\mathrm{L}}$, versus load force, $\mathrm{L}$ ) to this equation with $\alpha=1$, and $\alpha=0$, and using the measured $\mathrm{L}_{\mathrm{c}(\alpha)}$ and $\mathrm{a}_{0}$ and $\tau$ as fitting parameters, permits the determination of the appropriate model to use (JKR or DMT). In addition it allows the calculation of the interfacial adhesion energy, $\gamma$, as once either JKR or DMT have been selected $\mathrm{L}_{\mathrm{c}(\alpha)}$ can be used to calculate $\gamma$ as: 


$$
\begin{aligned}
& L_{c(1)}=-\frac{3}{2} \pi \gamma \cdot R \text { or } \\
& L_{c(0)}=-2 \pi \gamma \cdot R
\end{aligned}
$$

where $\mathrm{R}$ is the radius of the AFM tip

Also, once the interfacial adhesion energy, $\gamma$, is determined, the combined elastic modulus, $\mathrm{K}$, of the tip-sample can be determined by inserting the known values for $\mathrm{a}_{0}, \gamma$ and R:

$$
\begin{aligned}
& a_{0(J K R)}=\left(\frac{6 \pi \gamma \cdot R^{2}}{K}\right)^{1 / 3} \text { or } \\
& a_{0(D M T)}=\left(\frac{2 \pi \gamma \cdot R^{2}}{K}\right)^{1 / 3}
\end{aligned}
$$

As the Poisson ratio's of the AFM tip (single crystal silicon) and sample, and the Young's modulus of the AFM tip are known, the Young's modulus of the sample can thus be calculated as:

$$
K=\frac{4}{3}\left(\frac{\left(1-v_{t i p}{ }^{2}\right)}{E_{t i p}}+\frac{\left(1-v_{\text {samp }}{ }^{2}\right)}{E_{\text {samp }}}\right)^{-1}
$$

where $v_{\text {tip }}$ and $v_{\text {samp }}$ are the Poisson ratios of the tip and sample and $E_{\text {tip }}$ and $E_{\text {samp }}$ are the Youngs modulus of the tip and sample.

Finally, this process produces the interfacial shear stress, $\tau$, between the AFM tip and the sample, a value which is of particular interest to us. This parameter and the interfacial adhesion energy provide accurate determinations of the nature of the interactions between two surfaces.

In the case of the measurements reported below, these values report on the interactions between single silicon oxide filler particles mounted on the AFM cantilever (taking the place of the typical AFM tip) and the M9787 polymer samples. As previously stated the goal of this project is to relate modifications in this parameter to changes in the interaction between filler particles and the polymer matrix as a function of hydration, radiation exposure, and material aging.

Sample preparation Initial attempts at spin coating the unfilled M9787 silicone onto flat single-crystal silicon substrates met with failure. Without a high concentration of silicon oxide filler the polymer samples were too soft (almost a liquid) to image with AFM. Next, we coated the M9787 onto flat substrates of silicon oxide aerogels, reasoning that the increased surface area of the substrate would add stability to the polymer film. This was indeed the case and lateral force measurements were successfully obtained from 
these samples. However, the non-uniform roughness of the samples created a new problem with reproducibility. Although over microns the samples were flat, the nanoscale roughness of the polymer-coated aerogel surface "coupled in" to the lateral force measurements and different samples displayed widely different frictional coefficients. Recognizing that locally flat substrates were a necessity, we returned to the silicon substrates. We solved the softness issue initially by covalently attaching polymer "tethers" to the silicon substrates, spin-coating the M9787 on top of the modified substrates, and then cross-linking the M9787 to the tethers with exposure to ultraviolet light (UV) (Figure 10). This produced a rigid sample that closely approximated the filled silicones. While experimenting with the crosslinking process we discovered that the UV exposure of the M9787 (without the additional substrate preparation) was enough to produce the required rigidity, although the chemically treated samples were more rigid and displayed longer term stability.

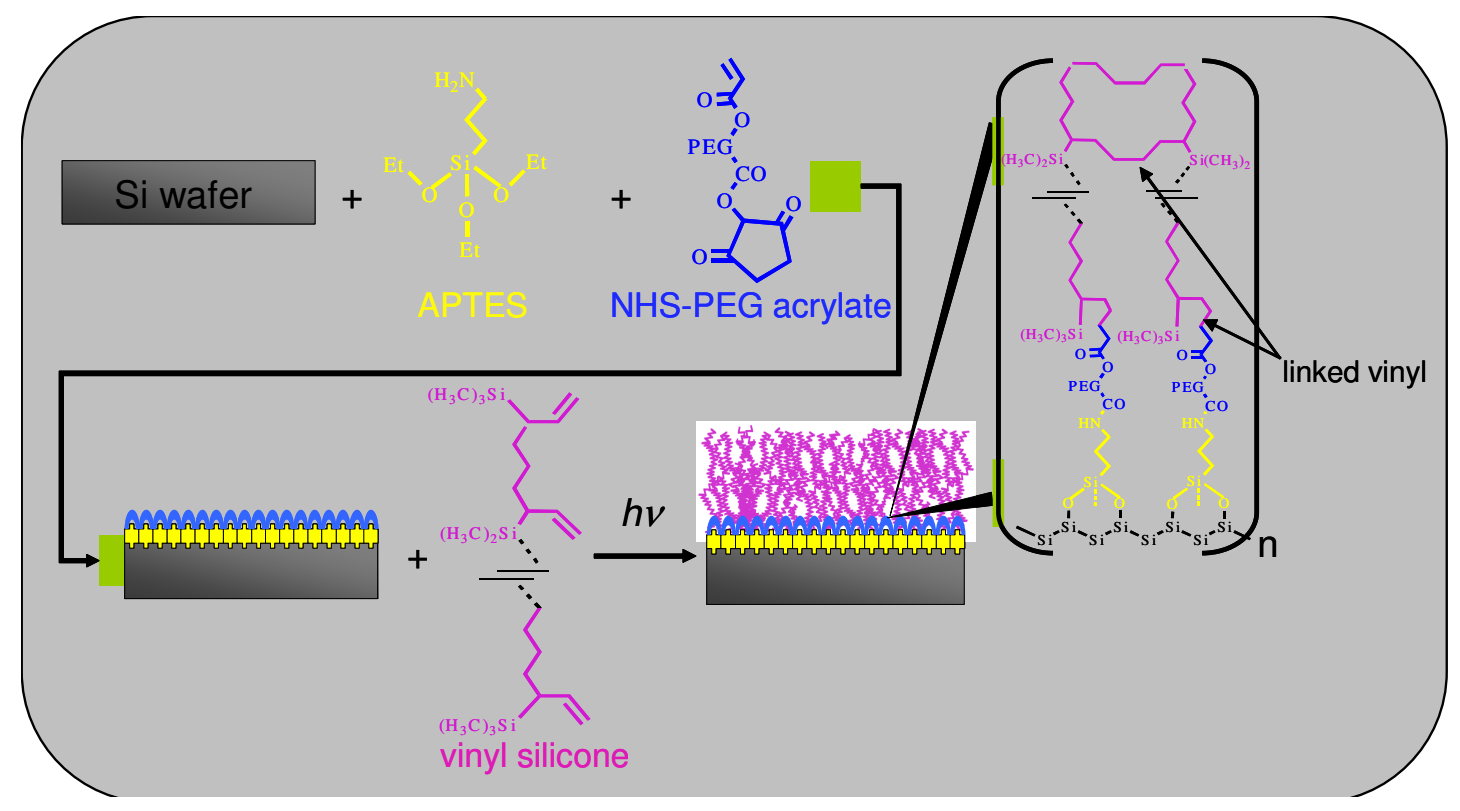

Figure 10. The schematic shown above outlines the process of crosslinking the M9787 polymer onto a silicon substrate. The chemical treatment of the substrate enables highly rigid thin films that do not require filler for robustness.

\section{Experiments:}

Results from imaging When this project initially began, we had questions concerning the nature of the material undergoing our analysis. We obtained samples of filled silicone pads (M9787) and imaged them with the AFM. In Figure 11, the lighter portions of the sample are higher. This image reveals two issues regarding direct analysis of this material. The first is that these polymer-filler composite materials contain large aggregates of filler particles as seen by the large variation in height across the sample. The second is that this material displays molding marks (the roughly $50 \mathrm{~nm}$ sized oval features) that prevent topographical images from revealing any details regarding the 
polymer-filler interaction by occluding nanoscale sample features. Due to these results we decided to produce our own samples and thus enable the reduction of the concentration of filler particles and variability due to the pad production. We imaged M9787 samples containing low concentrations of the silicon oxide filler produced in the method described above (Figure 12).

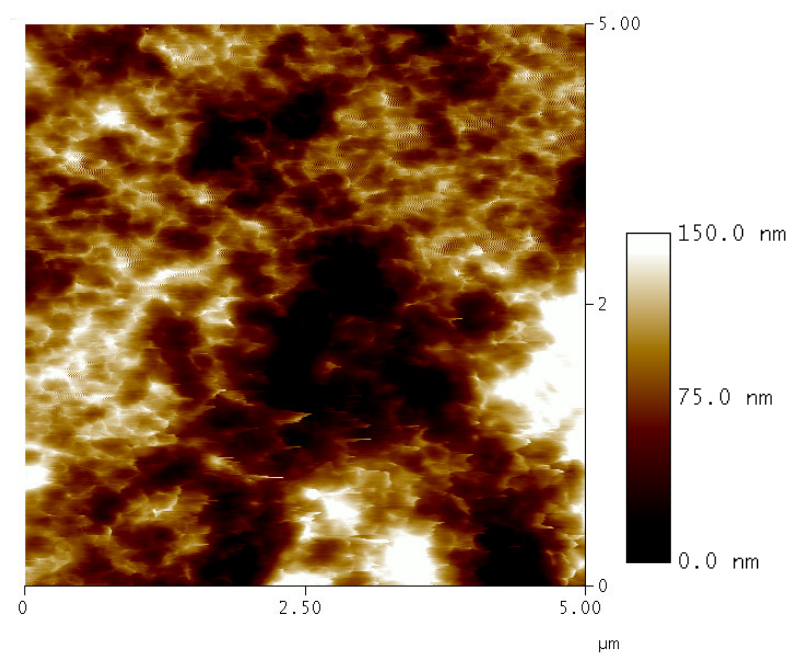

Figure 11. An AFM image of a M9787 silicone pad. The image shows the nonuniformity in the filler size and distribution within the polymer matrix.

Additionally, the small, roughly oval features are the result of the process of molding and pressure treating the pad.

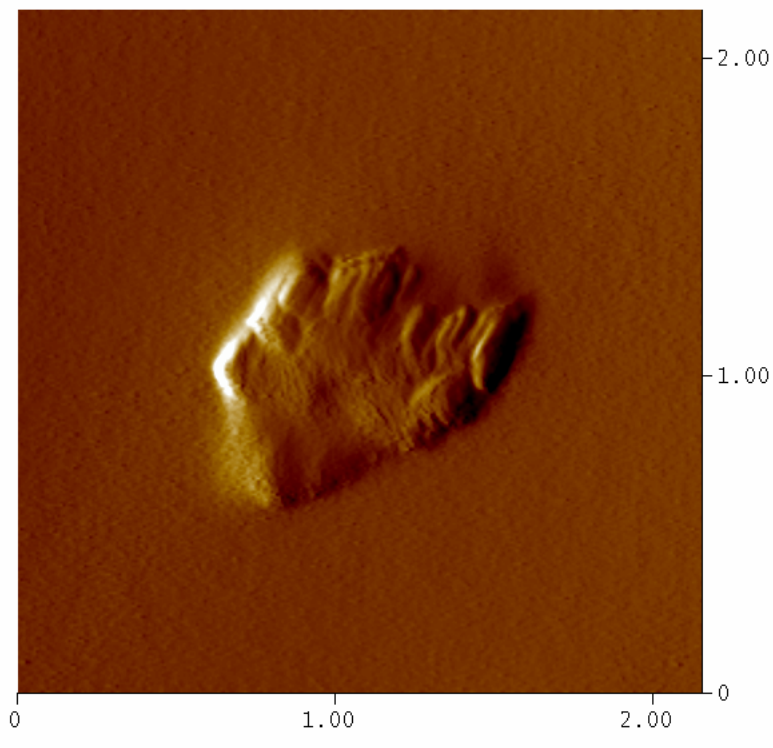

Figure 12. An AFM image of a M9787 thin film containing a silicon oxide particle aggregate. Even with long-term sonication of the polymer-filler solutions prior to spin-coating the solutions onto silicon substrates, single silicon oxide filler particles embedded in the polymer matrix could not be ohtained.

The images revealed that even at low filler concentrations the isolated particles were relatively large irregular aggregates. As an additional experiment we attempted to measure the elastic modulus of the polymer films as a function of distance from the filler particles, as earlier studies have indicated that the polymer matrix is modified at close proximity to the particle. These measurements are made by using a sharp AFM tip and indenting the sample at evenly spaced points across the sample. In the image shown in the inset of Figure 13 each pixel represents a single measurement of elastic modulus. Line scans taken from the three dimensional data are shown in Figure 13. Once again, the irregularly shaped filler aggregates prevented a reproducible measurement of the polymer 
elastic modulus near the particles, although the bulk modulus in the film was easily determined. The measured bulk elastic modulus closely matched earlier measurements of

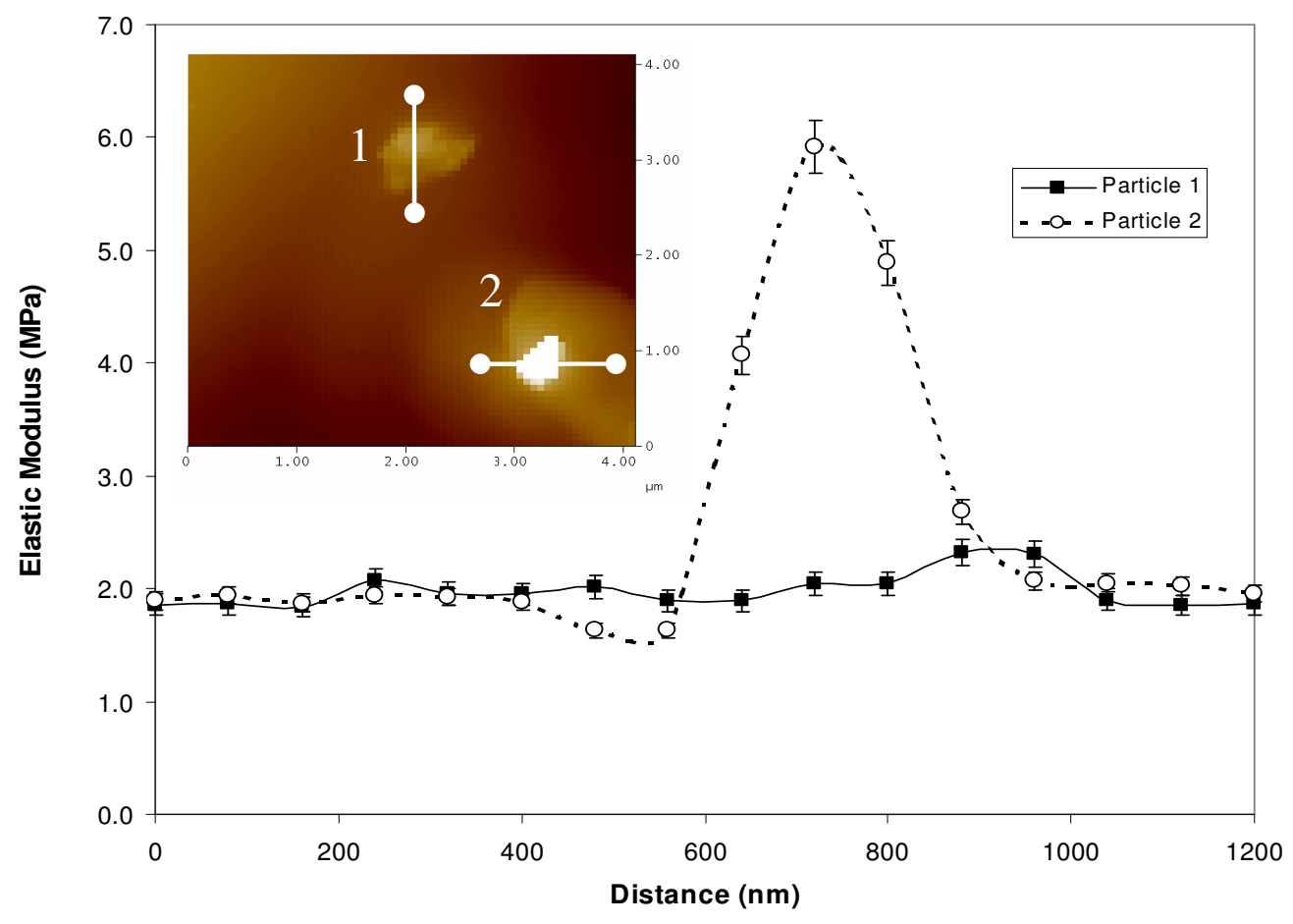

Figure 13. Elastic modulus measured across two silicon oxide filler particles embedded in an M9787 polymer film. In these measurements the irregular nature of the filler particles resulted in two widely varying values for the modulus close to the filler particles. The modulus in the bulk unfilled material was $\sim 2.0 \mathrm{MPa}$, a value similar to earlier measurements. The inset shows the location of the two line scans.

similarly prepared M9787 films, $2.0 \mathrm{MPa}$, and increased dramatically near Particle 1 but not Particle 2. This effect likely reflected probing the hard silicon oxide material of Particle 1 rather than the polymer film associated with the particle, as it could be seen from the height image that Particle 1 extended some distance from the surface of the film. Particle 2 was mostly buried under the surface of the polymer film and made no contribution to the measured elastic modulus, even though the polymer film was surely much thinner above the particle in comparison to the bulk film. It appeared that the film thickness had little effect on the bulk elastic modulus. This is to be expected when the indenting tip is much smaller than the film thickness, as it is in our case. We tested this by preparing films of various thicknesses and repeating the experiment. We found that in all cases the elastic modulus of the film was independent of the thickness (in contrast to this, the measured interfacial shear stress, $\tau$, was directly dependent on the thickness of the polymer film, as we report in the following section).

In order to remove the effects of the irregularity in shape of the filler particles, one micron spherical silicon oxide beads were purchased and M9787 polymer solutions were prepared using the beads in place of the regular silicon oxide filler. Prior to repeating the indentation experiments, we imaged the samples first to measure the topography of the polymer films containing the embedded silicon oxide beads, and then 
using a form of tapping mode imaging which generates contrast based on the mechanical characteristics of the sample (Figure 14). This kind of imaging allows qualitative determinations of the elastic modulus of thin films. From Figure 14A it can be seen that the film modulus varies dramatically in the region of the embedded particles, creating rings of influence extending outward from the silicon oxide beads. When the influence from one bead intersects with another complex interactions in the film modulus are produced. This result was rather surprising, as interactions between the silicon oxide particles and the polymer matrix are only expected to extend outward from the particle a few polymer lengths ( $<<1.0$ micron). We now believe that this long range effect is not due to a specific interaction between the silicon oxide surface of the filler particle and the polymer matrix but rather an artifact from the preparation of the film in which the polymer film density is modified by the presence of the particles. It remains to be seen whether or not other film preparation methods such as molding or physical spreading, rather than our current spin-coating technique, produce similar effects. If so, these density variations could have significant consequences on the aging of the material and would need to be included in computational models that predict long-term material behavior.

\section{Force Measurements}

In order to further examine the interactions between silicon oxide particles and the M9787 polymer films, we performed normal and lateral force experiments using a single silicon oxide filler particle mounted on an AFM cantilever in order to obtain; the correct physical model to use (JKR or DMT), the interfacial adhesion energy, $\gamma$, and the shear stress, $\tau$. Samples were prepared by spin-coating the M9787 polymer at various thicknesses onto flat single crystal silicon substrates and then cross linking the polymer with ultraviolet light as described above. The cantilevers were calibrated to obtain the normal and lateral detector sensitivities and the normal and torsional spring constants prior to each experiment. The AFM is encased in an environmental chamber that permits
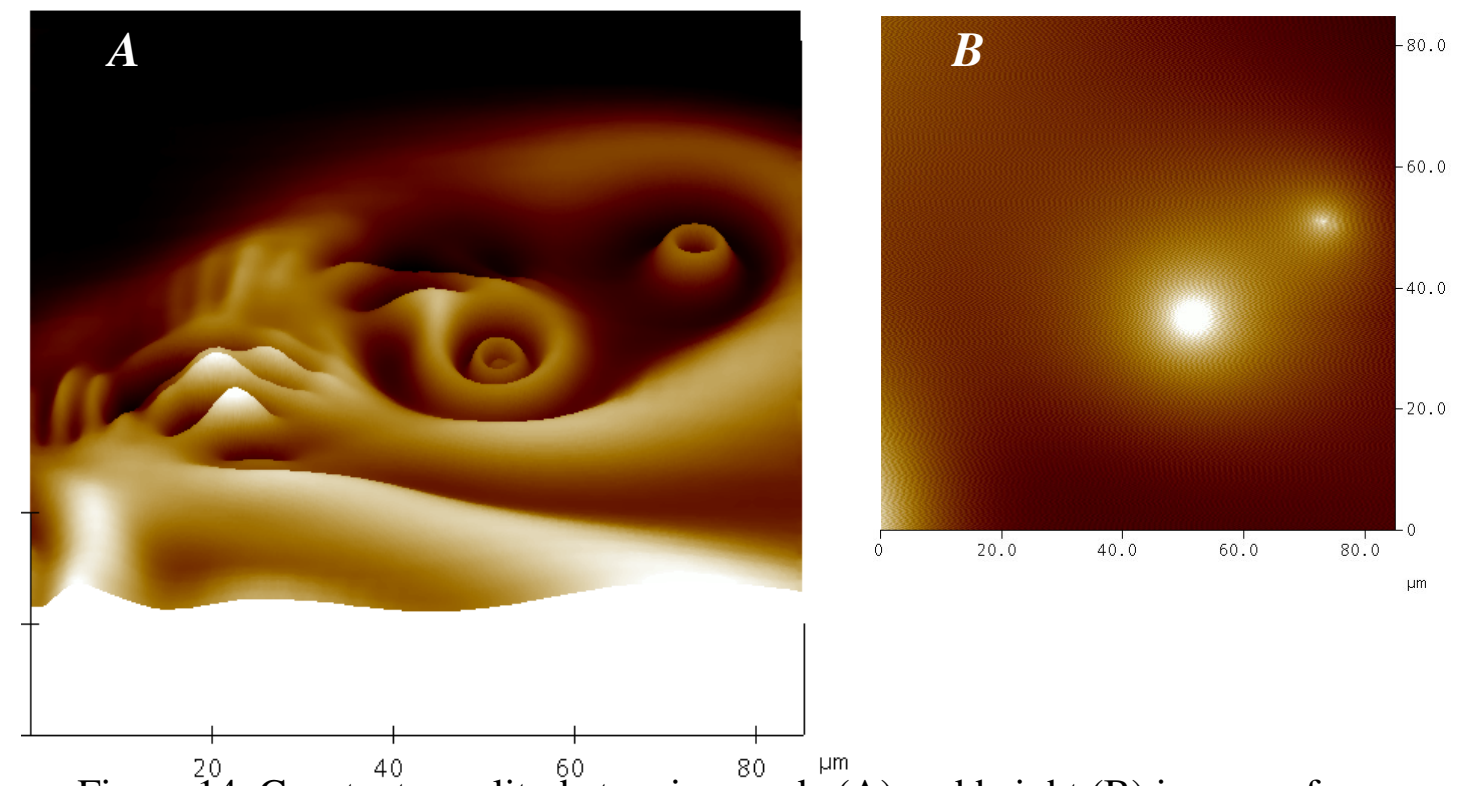

Figure 14. Constant-amplitude tapping mode (A) and height (B) images of an M9787 polymer film with embedded silica beads. The tapping mode image shows variations in the modulus of the polymer matrix generated by the presence of the silica beads. 
the humidity to be reduced down to $\sim 0.04 \%$ relative humidity. Dry nitrogen is flowed through the chamber for at least 24 hours before each experiment and the cantilevers are cleaned with UV-ozone to remove water or any organic contaminants.

For our initial control experiment, we wanted to determine if the measured lateral force on the polymer films was dependent on the rate at which we produced the friction loops. We performed an experiment where we measured the lateral force with the applied load held constant while varying the scan rate (for a regular lateral force measurement, the lateral force is measured at a constant scan rate while the applied load is increased). We found strong rate dependencies for all loads at scan rates below $\sim 12.2 \mathrm{um} / \mathrm{second}$ (Figure 15). That is at rates slower than this, the lateral force increased with scan rate.

Higher loading forces appeared to be slightly less rate dependent, for example, at a constant applied load of $3 \mathrm{~V}$, the lateral force did not increase once the scan rate had reached $\sim 8 \mathrm{um} / \mathrm{second}$. The rate dependence is likely due to the deformation of the polymer film around the silicon oxide bead when the bead is in contact with the film. At lower scan rates the bead pushes the deformation in front of it, and this contributes to the measured lateral force. At higher scan rates the bead skips over the deformation in the film and so the measured lateral force is due solely to interactions between the bead and the polymer film. To test this hypothesis we repeated the measurement on a flat piece of silicon with no polymer film. As expected the lateral force showed only a slight dependence on the scan rate. From these results we resolved to make all lateral force measurements at scan rates above $12.2 \mathrm{um} / \mathrm{second}$ and at loading rates higher than 48.6 $\mathrm{nN}$.

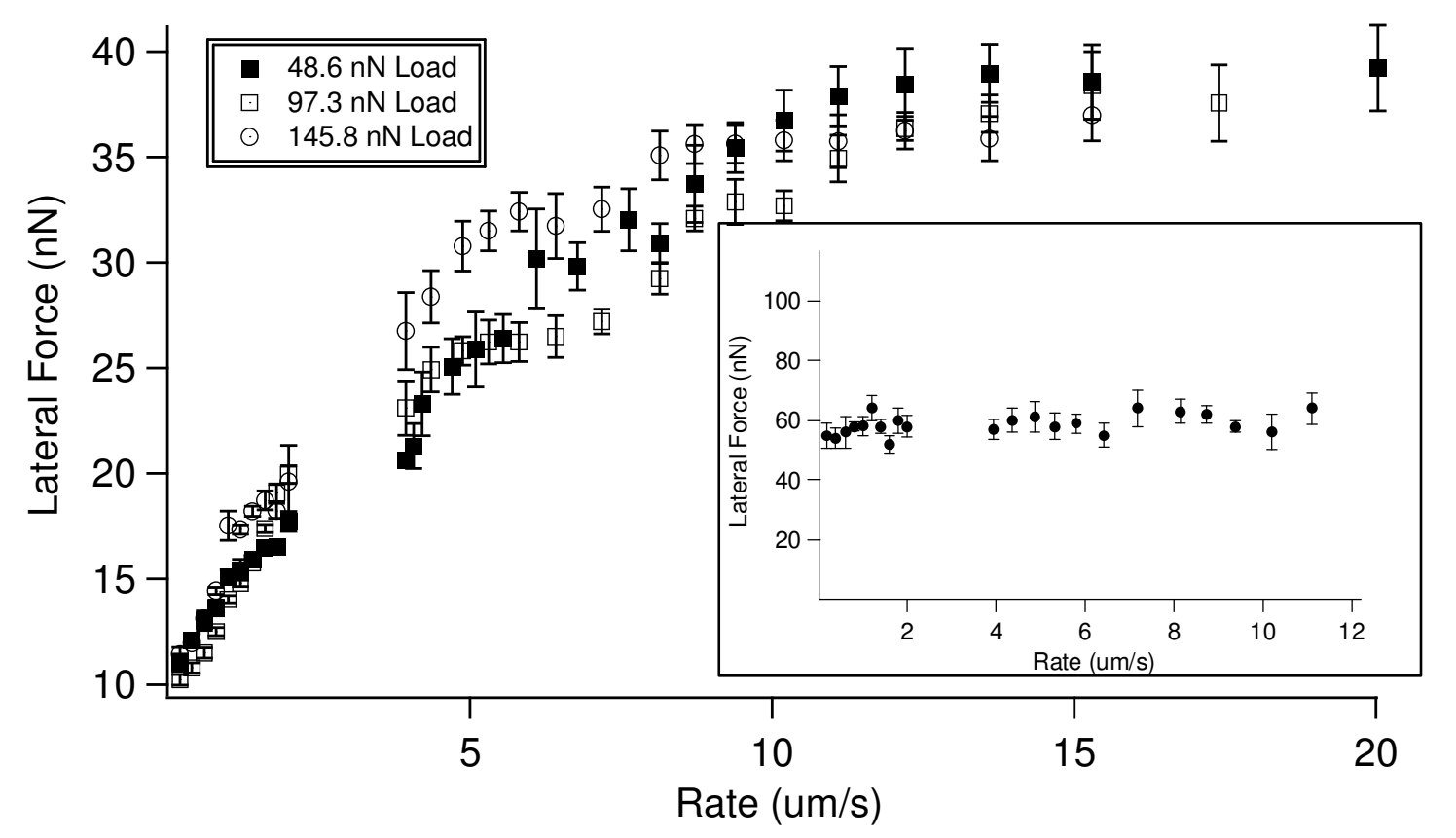

Figure 15. Lateral force as a function of scan rate. This experiment was performed in order to determine the optimum scan rate for making the lateral force measurements. From these results we determined that at scan rates above 12.2. um/second, and applied loads greater than $48.6 \mathrm{nN}$ the measured lateral force is independent of scan rate. The inset shows a measurement performed on a bare silicon substrate where almost no rate dependence was observed. 


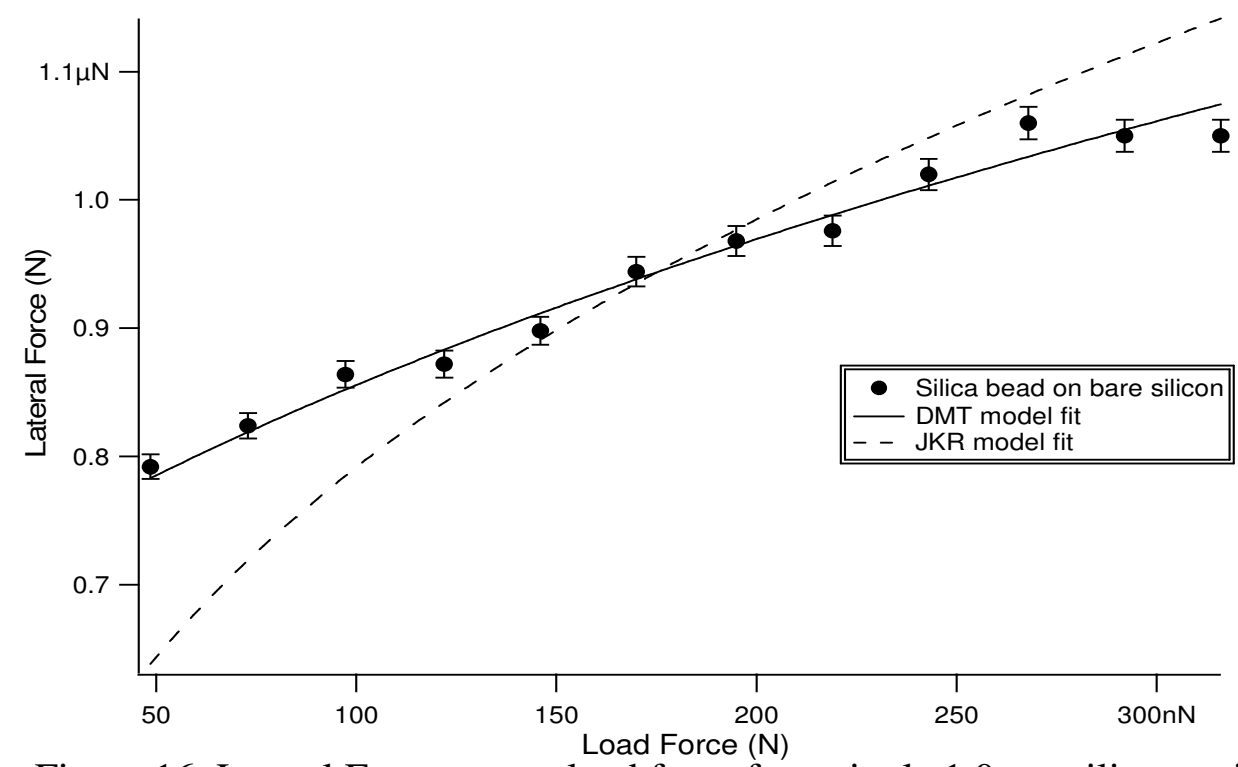

Figure 16. Lateral Force versus load force for a single $1.0 \mathrm{um}$ silicon oxide bead on single crystal silicon. Both the DMT and JKR models were fit to the data with the contact patch, $\mathrm{a}_{0}$, and the shear stress, $\tau$, as fitting parameters. The DMT model fit the data best, generating a contact patch of $\sim 0.04 \mathrm{um}$, and a shear stress of 1.3 MPa.

After determining the optimum scan rates, we began the lateral force measurements by first measuring adhesion between the silicon oxide bead mounted on the AFM cantilever and a bare silicon surface (the adhesion force is required for the application of the physical models to the lateral force data). Then the lateral force as a function of load was measured and the two physical models (JKR and DMT) were fit to the data. For these

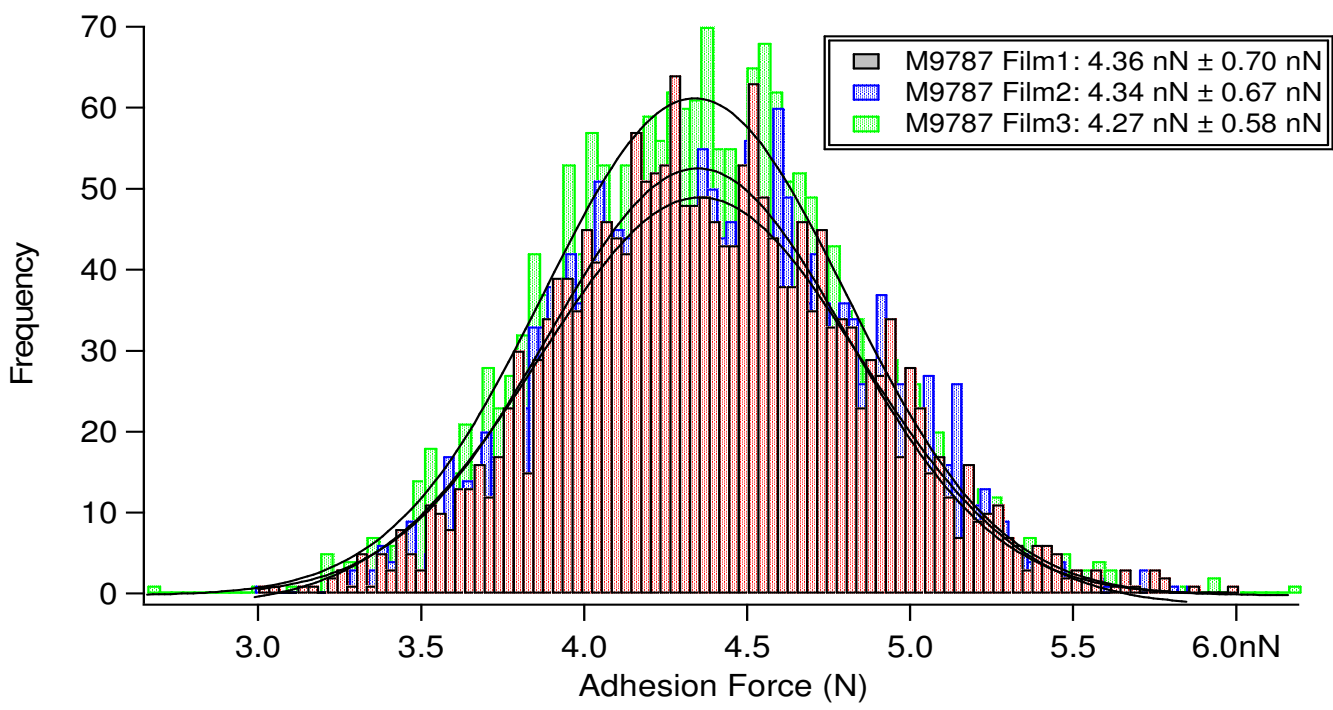

Figure 17. Histograms of adhesion force measurements made between silica beads and three different polymer films. 
measurements, ten friction loops are generated at each load force, and the values are averaged. The error bars in the graphs are the standard deviations. As seen in Figure 16, the DMT model fit much better to the bead on bare silicon data. This is to be expected as the DMT model applies in cases where the substrate material is rigid. The model fits revealed a contact radius of $\sim 0.04 \mathrm{um}$, in keeping with estimations for a 1.0 micron bead in contact with a hard substrate, and a shear strength of $1.3 \mathrm{MPa}$, an appropriate range for interactions between silicon/silicon oxide.

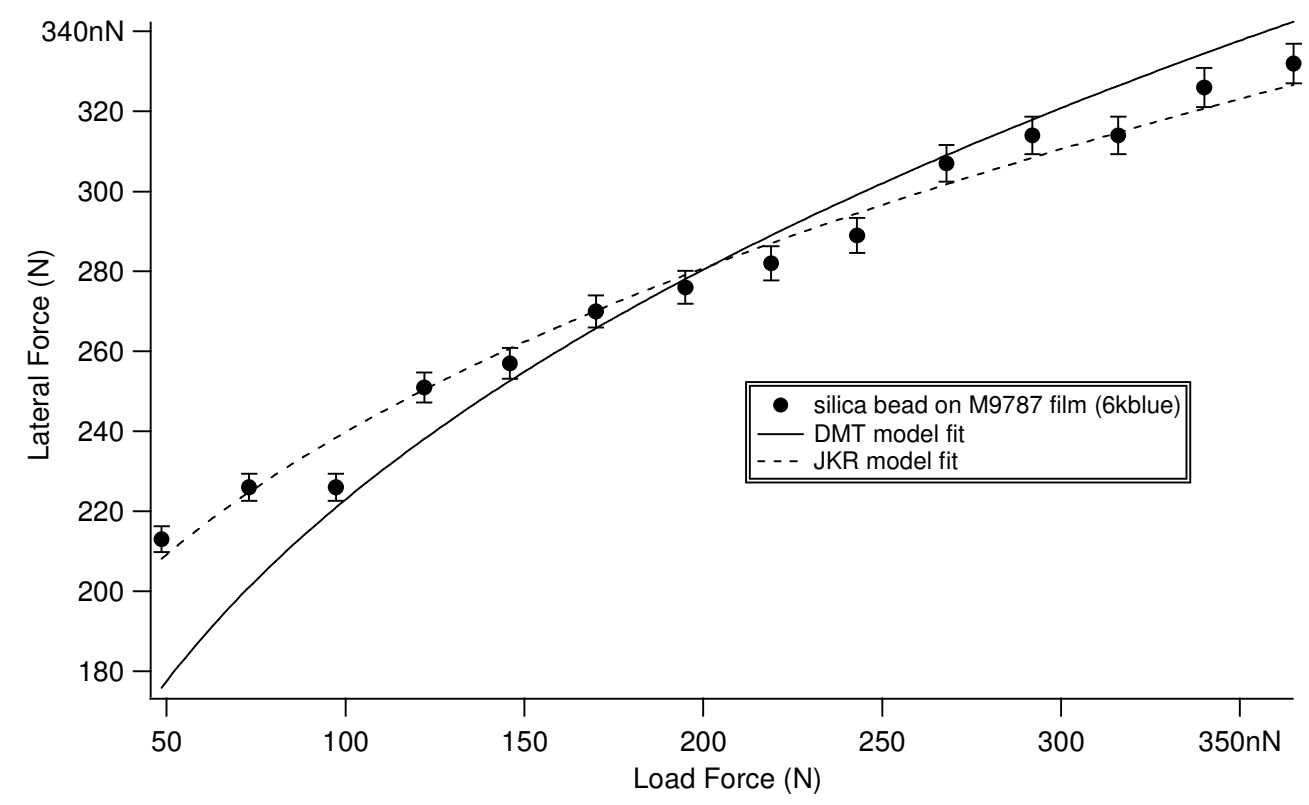

Figure 18. Lateral force vs. applied load for a 1.0 um silica bead on M9787 polymer film. Both the DMT and JKR models were fit to the data with the contact patch, $\mathrm{a}_{0}$, and the shear stress, $\tau$, as fitting parameters. The JKR model fit the data best, generating a contact patch of $\sim 0.05 \mathrm{um}$, and a shear stress of $\sim 0.12 \mathrm{MPa}$.

In a similar manner the adhesion force and then the lateral force as a function of applied load were measured between a 1.0 um silica bead and an M9787 polymer film. The adhesion data from three different polymer films is shown in Figure 17. Large numbers of pull-off measurements are made (the tip is placed in contact with the substrate and then pulled off while monitoring the deflection of the cantilever).

Histograms of the adhesion force versus frequency are generated and the data is fit with a Gaussian. The adhesive forces between the silica bead and the polymer averaged $\sim 4.32 \pm$ $0.65 \mathrm{nN}$.

In this case, the JKR model rather than the DMT model fit the lateral force data best, as shown in Figure 18. From the model fit, the contact radius was determined to be $\sim 0.05 \mathrm{um}$, in keeping with the expected contact radius for a $1.0 \mathrm{um}$ bead in contact with a compliant film. The shear strength was much lower for the polymer film in comparison with the bare silicon substrate, ( 0.12 MPa versus $1.32 \mathrm{MPa}$ on bare silicon).

In order to determine one of the possible sources of error in the shear strength determination for a polymer sample, we repeated the measurements three times at three different areas of the sample and fit the data as before (Figure 19). Although for all three areas the lateral force at zero applied load differed- possibly due to slight differences in 
the contact patch between the silica bead and the polymer film- the measured shear strengths were consistently $\sim 0.125 \pm .01 \mathrm{MPa}$.

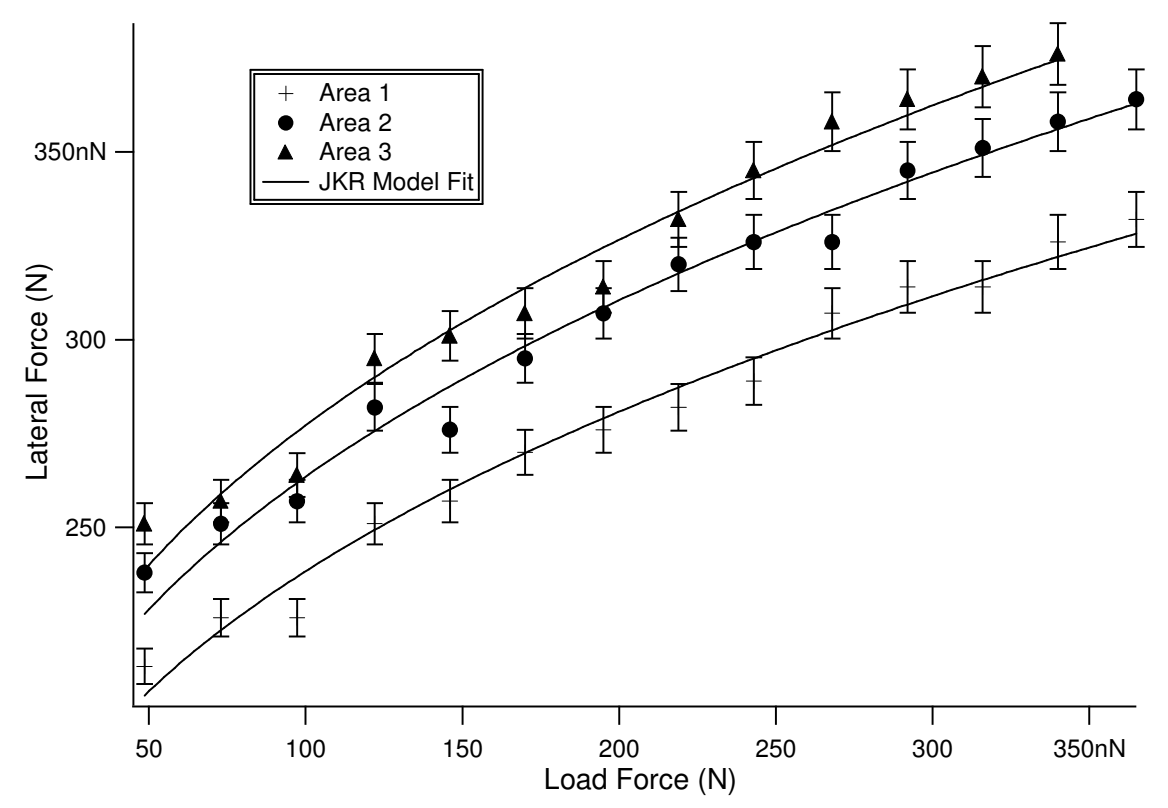

Figure 19. Lateral force vs applied load for a 1.0 um bead on an M9787 polymer film- three different areas. All areas showed similar shear moduli and contact patch radii.

Finally, the effects on the mechanical characteristics of the polymer films due to changes in thickness were evaluated. As discussed earlier, the polymer-coated silicon substrate is a reasonable model for a filler particle surrounded by the polymer matrix. Thus varying the thickness of the polymer film between the silica bead on the cantilever and the silicon substrate is a method whereby the nature of the polymer film between two filler particles in a real filled polymer system can be investigated. Three polymer samples were prepared at different thicknesses by spin-coating the polymer solution onto silicon substrates at different spin rates. A portion of each sample was masked off and the remaining areas were crosslinked with ultraviolet light. After removing the mask the samples were rinsed in toluene to remove the non-crosslinked polymer and a DekTak stylus profiler was used to characterize the thickness of the polymer film at the film interface. The adhesion forces between silica beads and the polymer films were measured and the lateral force versus loading force obtained. Finally, model fits to the lateral force were made as described earlier. The results from these measurements are shown in Figure 20. Interestingly, although the adhesion energy and elastic modulus did not vary dramatically with polymer film thickness, the shear strength more than doubled as the film thickness decreased. This behavior may be partially ascribed to a greater degree of interaction between the silica bead and the silicon substrate as the contact patch between the bead and film becomes larger than the polymer film thickness. Furthermore, the increase in shear strength may in part be due to increased ordering of the polymer chains at the silicon oxide interface as has been predicted in other studies. Finally, effects due to density variations in the polymer films (as a result of the spin-coating method) may also play a role in the increase in shear strength. Clearly, additional experiments are necessary 
to identify the specific mechanisms responsible for changes in the mechanical characteristics of silicone films. The results from all current experiments are tabulated in Table 1.

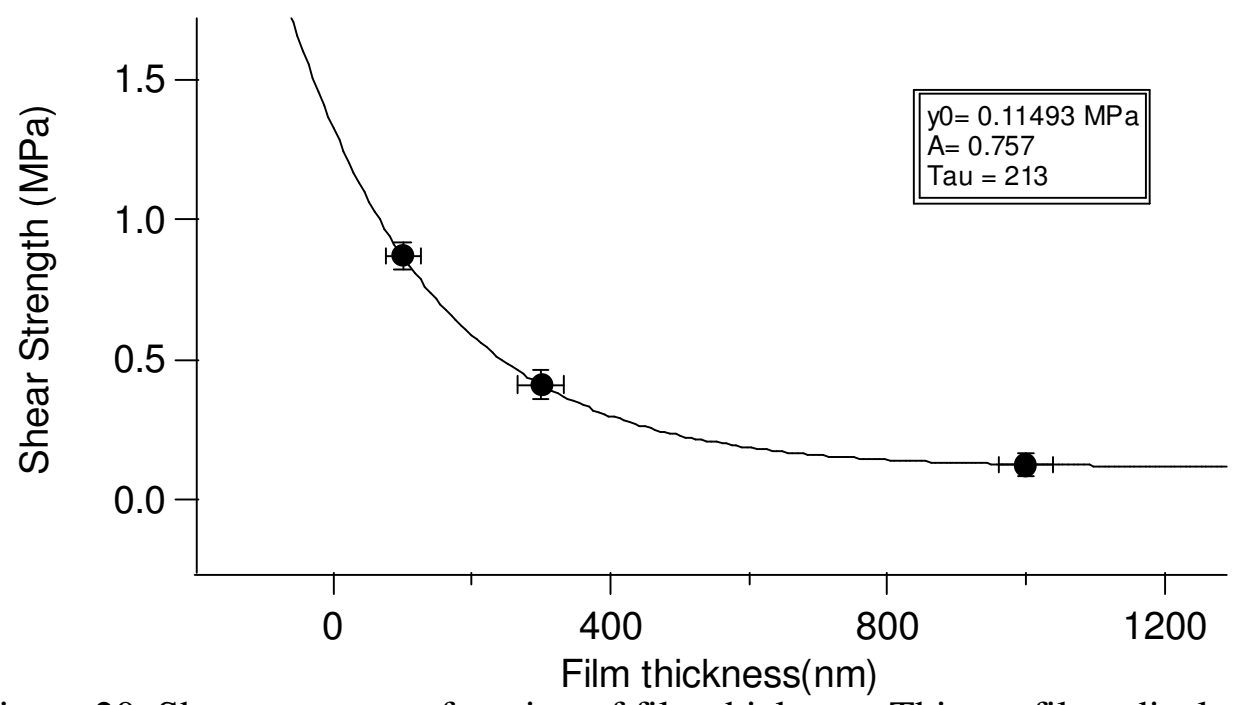

Figure 20. Shear stress as a function of film thickness. Thinner films displayed higher shear strengths. This may be due to increased ordering of the polymer film near the silicon oxide interface, an increased degree of interaction between the silica bead and the silicon substrate, or possibly variations in polymer density due to variations in the sample preparation. The $\mathrm{x}$ and $\mathrm{y}$ error bars are due to errors in the model fit to the data, and errors in the determination of film thickness, respectively.

\begin{tabular}{|l|l|l|l|l|l|}
\hline Substrate & Model & $\begin{array}{l}\text { Adhesion } \\
\text { energy }(\gamma, \\
\mathrm{J} / \mathrm{m})\end{array}$ & $\begin{array}{l}\text { Contact } \\
\text { radius } \\
\left(\mathrm{a}_{0}, \mathrm{um}\right)\end{array}$ & $\begin{array}{l}\text { Elastic } \\
\text { modulus } \\
(\mathrm{E}, \\
\mathrm{MPa})\end{array}$ & $\begin{array}{l}\text { Shear } \\
\text { strength } \\
(\tau, \mathrm{MPa})\end{array}$ \\
\hline Bare silicon & DMT & $0.49 \mathrm{e}-3$ & 0.041 & NA & 1.32 \\
\hline M9787 Film 1 & JKR & $1.42 \mathrm{e}-3$ & 0.055 & 1.98 & 0.119 \\
\hline M9787 Film 1 & JKR & $1.38 \mathrm{e}-3$ & 0.049 & 2.11 & 0.126 \\
\hline M9787 Film 1 & JKR & $1.39 \mathrm{e}-3$ & 0.051 & 2.08 & 0.130 \\
\hline M9787 Film 2 & JKR & $1.49 \mathrm{e}-3$ & 0.045 & 1.95 & 0.354 \\
\hline M9787 Film 3 & JKR & $1.52 \mathrm{e}-3$ & 0.039 & 1.92 & 0.872 \\
\hline
\end{tabular}

Table 1. Interfacial characteristics for the adhesive and frictional interactions between one um silica beads attached to AFM cantilevers and various substrates. The three polymer films were of decreasing thickness $(1,2,3)$ 


\section{Future Work:}

We have begun the process of examining filled polymer systems both with AFM imaging and with the measurements of adhesion and lateral forces between model filler particles and silicone films. Although the work thus far has served mainly to validate the method and lay the groundwork for future experiments we have begun to make useful observations regarding the mechanical characteristics of these systems. Future work will involve examining in depth how modifications to the mechanical characteristics take place, specifically, how environmental factors such as hydration/dehydration, radiation exposure, and aging influence mechanical stability. The main goal behind the acquisition of these data is to feed coarse-grained polymer dynamic simulations for the construction of a predictive polymer model. Once the initial model is in place, it will be refined by comparing it against empirical measurements of the behavior of specialized test polymer materials under various conditions. These test materials will consist of silicone polymers that are filled with the same silica beads that are used to form the test probes described in the present work. When the model has been successfully refined with respect to the test material, it will then undergo mathematical generalization to account for the highly variable nature of the fumed silica fillers that are used in practical polymer materials.

\section{References}

1. Binnig, G.; Quate, C. F.; Gerber, C., Atomic Force Microscope. Physical Review Letters 1986, 56, (9), 930-933.

2. $\quad$ Langry, K. C.; Ratto, T. V.; Rudd, R. E.; McElfresh, M. W., The AFM measured force required to rupture the dithiolate linkage of thioctic acid to gold is less than the rupture force of a simple gold-alkyl thiolate bond. Langmuir 2005, 21, (26), 1206412067.

3. Ratto, T. V.; Langry, K. C.; Rudd, R. E.; Balhorn, R. L.; Allen, M. J.; McElfresh, M. W., Force spectroscopy of the double-tethered concanavalin-A mannose bond. Biophysical Journal 2004, 86, (4), 2430-2437.

4. $\quad$ Ratto, T. V.; Langry, K. C.; Rudd, R. E.; Balhorn, R. L.; McElfresh, M. W., Force spectroscopy of single-molecule adhesive interactions in three polymer-tethered multivalent systems: Proteincarbohydrate, thiol/thiolate-gold, and shal-protein bond pairs. Abstracts Of Papers Of The American Chemical Society 2005, 229, U633-U634.

5. $\quad$ Ratto, T. V.; Rudd, R. E.; Langry, K. C.; Balhorn, R. L.; McElfresh, M. W., Nonlinearly additive forces in multivalent ligand binding to a single protein revealed with force spectroscopy. Langmuir 2006, 22, (4), 1749-1757.

6. Sulchek, T. A.; Friddle, R. W.; Langry, K.; Lau, E. Y.; Albrecht, H.; Ratto, T. V.; DeNardo, S. J.; Colvin, M. E.; Noy, A., Dynamic force spectroscopy of parallel individual Mucin1-antibody bonds. Proceedings Of The National Academy Of Sciences Of The United States Of America 2005, 102, (46), 16638-16643.

7. Vezenov, D. V.; Noy, A.; Ashby, P., Chemical force microscopy: probing chemical origin of interfacial forces and adhesion. Journal Of Adhesion Science And Technology 2005, 19, (3-5), 313-364.

8. $\quad$ Green, C. P.; Lioe, H.; Cleveland, J. P.; Proksch, R.; Mulvaney, P.; Sader, J. E., Normal and torsional spring constants of atomic force microscope cantilevers. Review Of Scientific Instruments 2004, 75, (6), 1988-1996. 
9. Butt, H. J.; Jaschke, M., Calculation Of Thermal Noise In Atomic-Force Microscopy. Nanotechnology 1995, 6, (1), 1-7.

10. Jaschke, M.; Butt, H. J.; Manne, S.; Gaub, H. E.; Hasemann, O.; Krimphove, F.;

Wolff, E. K., The atomic force microscope as a tool to study and manipulate local surface properties. Biosensors \& Bioelectronics 1996, 11, (6-7), 601-612.

11. Carpick, R. W.; Ogletree, D. F.; Salmeron, M., Friction, adhesion, contact area and load measurements of nanometer-sized contacts using friction force microscopy. Abstracts Of Papers Of The American Chemical Society 1997, 213, 8-COLL.

12. Enachescu, M.; van den Oetelaar, R. J. A.; Carpick, R. W.; Ogletree, D. F.; Flipse, C. F. J.; Salmeron, M., Observation of proportionality between friction and contact area at the nanometer scale. Tribology Letters 1999, 7, (2-3), 73-78.

13. Etsion, I.; Levinson, O.; Halperin, G.; Varenberg, M., Experimental investigation of the elastic-plastic contact area and static friction of a sphere on flat. Journal of Tribology-Transactions Of The Asme 2005, 127, (1), 47-50.

14. Liu, E.; Blanpain, B.; Celis, J. P.; Roos, J. R., Comparative study between macrotribology and nanotribology. Journal Of Applied Physics 1998, 84, (9), 4859-4865.

15. Tomasetti, E.; Legras, R.; Nysten, B., Quantitative approach towards the measurement of polypropylene/(ethylene-propylene) copolymer blends surface elastic properties by AFM. Nanotechnology 1998, 9, (4), 305-315.

16. Cain, R. G.; Biggs, S.; Page, N. W., Force calibration in lateral force microscopy. Journal Of Colloid And Interface Science 2000, 227, (1), 55-65.

17. Green, C. P.; Sader, J. E., Frequency response of cantilever beams immersed in viscous fluids near a solid surface with applications to the atomic force microscope. Journal Of Applied Physics 2005, 98, (11).

18. Sader, J. E.; Pacifico, J.; Green, C. P.; Mulvaney, P., General scaling law for stiffness measurement of small bodies with applications to the atomic force microscope. Journal Of Applied Physics 2005, 97, (12).

19. Varenberg, M.; Etsion, I.; Halperin, G., An improved wedge calibration method for lateral force in atomic force microscopy. Review Of Scientific Instruments 2003, 74, (7), 3362-3367.

20. Varenberg, M.; Etsion, I.; Halperin, G., Crosstalk problems in scanning-by-probe atomic force microscopy. Review Of Scientific Instruments 2003, 74, (7), 3569-3571. 21. Israelachvili, J.; Maeda, N.; Rosenberg, K. J.; Akbulut, M., Effects of subfingstrom (pico-scale) structure of surfaces on adhesion, friction, and bulk mechanical properties. Journal Of Materials Research 2005, 20, (8), 1952-1972.

22. Carpick, R. W.; Ogletree, D. F.; Salmeron, M., A general equation for fitting contact area and friction vs load measurements. Journal Of Colloid And Interface Science 1999, 211, (2), 395-400. 


\section{Appendix 1: Normal -mode calibration}

The procedure for calibrating the AFM for normal mode force measurements is as follows:

\section{Measurements:}

1. mount a flat substrate in the AFM

- clean silicon wafers or freshly-cleaved mica or graphite work well

2. mount cantilever to be calibrated in the AFM

3. position laser for strong signal with compact spot size

- signal measured with the AFM's $A+B$ meter

- spot inspected by inserting a small piece of paper in the beam

4. zero the $A-B$ signal

- achieved by positioning the photodetector in our instrument

5. do a coarse approach

6. do auto approach to sample

7. go to force curves

8. record $A-B$ (deflection) data during force curves

- note ramp size and rate

9. withdraw from the substrate

- by several microns, beyond the range of surface forces

10. send $A-B$ (deflection) data into spectrum analyzer and collect thermal spectrum

- $A-B$ signal must be zeroed well enough to make use of this sensitivity

- data rate must be comfortably more than double the cantilever resonant frequency to be able to characterize the resonant peak

- enough data should be acquired to allow a clean power spectrum to be generated (we usually record a time series of several million data points over several 10 s of seconds)

\section{Analysis}

The data analysis (which is automated in Igor Pro) involves the following steps:

1. from step eight above measure the slope of the contact portion of the force curve

- a smallish deflection range should be used to avoid detector nonlinearities

- $\quad$ since the substrate was rigid, the calibrated slope should be 1; thus the reciprocal of this slope is the deflection calibration factor $s$

2. calculate the power spectral density (PSD) of the noise data from step ten above

3. identify the peak corresponding to the fundamental resonant mode

- the manufacturer's specs are usually enough to figure out which peak is which

- beware of aliasing: if you suspect that a peak is not in the right place, try increasing your acquisition rate 
- also beware of electronic noise (usually a very sharp peak) or other vibrational modes

4. fit a Lorentizian (with baseline) to the peak

- the functional form is:

$$
P(f)=\frac{A}{\left(f-f_{0}\right)+B}
$$

where $\mathrm{A}$ and $\mathrm{B}$ are fitting parameters

5. determine the area under the peak from the fit parameters

$$
P=\pi \frac{A}{\sqrt{B}}
$$

6. estimate the ambient temperature $T$ of the cantilever

- ours runs at about $305 \mathrm{~K}$

7. apply the thermal calibration formula to determine $k$

The PSD is easily obtained using a spectrum analyzer that can measure up to $100 \mathrm{kHz}$ (the majority of cantilevers have normal mode resonant frequencies $<100 \mathrm{kHz}$ ) and this process is relatively quick and easy once the equipment is set up, each determination of the cantilever spring constant taking about five minutes. Once the spring constant, $\mathrm{k}_{\mathrm{z}}$, and the normal photodetector sensitivity, $\zeta_{\mathrm{L}}$, are determined the adhesion force between an AFM tip and a sample surface can be measured by producing a force curve. This is accomplished by moving the tip into contact with the surface and then retracting the tip while measuring the deflection of the cantilever. 